\title{
Relación entre el quiste hepático simple múltiple y la litiasis vesicular
}

Hubert James Mendoza Rojas ${ }^{1, a}$

\section{RESUMEN}

Objetivo: Conocer si hay relación entre el quiste hepático simple múltiple y la litiasis vesicular.

Materiales y métodos: Se realizó un estudio observacional, retrospectivo y transversal. Con un muestreo no probabilístico por conveniencia de tipo accidental. El tamaño de la muestra fue de 50 pacientes, con diagnóstico por imágenes de quiste hepático simple.

Resultados: El grupo etario más frecuente fue entre 61-70 años (32\%). El género femenino fue el más frecuente con 92 \%. Los quistes hepáticos simples tuvieron una media de 87,58 cc de volumen, con un mínimo de 0,16 cc y un máximo de 1255 cc. Un 48 \% de los quistes fueron múltiples. El Coeficiente de correlación lineal de Pearson para las variables edad y volumen del quiste fue de 0,035. Se localizaron en un 55,4\% en el lóbulo derecho, siendo el segmento II el más frecuente (17,3\%). El $56 \%$ de los quistes hepáticos simples se encontraron en hígados normales, seguido por un 36 \% en hígados con esteatosis. El $83,78 \%$ no presentaron litiasis vesicular. No se encontró dilatación de vías biliares en el $100 \%$ de los casos. No hubo relación entre el quiste hepático simple múltiple y la presencia de litiasis 0,828 $(p<0,05)$.

Conclusiones: No se evidenció una relación entre la presencia del quiste hepático simple múltiple y la litiasis vesicular.

Palabras claves: Litiasis; hígado; cálculos biliares; quistes (Fuente: Decs BIREME).

\section{Relationship between multiple simple hepatic cysts and vesicular lithiasis}

\section{ABSTRACT}

Objective: To know if there is a relationship between multiple simple hepatic cysts and vesicular lithiasis.

Materials and methods: An observational, retrospective and cross-sectional study was performed with a non-probability convenience (accidental) sampling. The sample size consisted of 50 patients diagnosed with simple hepatic cysts by ultrasound.

Results: The most frequent age group was between 61 and 70 years (32\%), and the female gender was the most common one (92\%). The simple hepatic cysts had a mean volume of $87.58 \mathrm{cc}$, with a minimum volume of $0.16 \mathrm{cc}$ and a maximum of 1255 cc. Multiple cysts accounted for $48 \%$ of all cysts. Pearson's linear correlation coefficient for variables age and cyst volume was 0.035 . Fifty-five point four percent (55.4\%) of the cysts were located in the right lobe, mostly on segment II (17.3\%). Fifty-six percent (56 \%) of simple hepatic cysts were found in normal livers, followed by $36 \%$ in livers with steatosis. Eightythree point seven eight percent $(83.78 \%$ ) did not show vesicular lithiasis. No dilation of bile ducts was found in $100 \%$ of the cases. There was no relationship between multiple simple hepatic cysts and lithiasis $(0.828)(p<0.05)$.

Conclusions: There was no evidence of a relationship between multiple simple hepatic cysts and vesicular lithiasis.

Keywords: Lithiasis; liver; gallstones; cysts (Source: MeSH NLM).

1. Hospital III de Emergencias Grau (EsSalud). Lima, Perú.

a. Cirujano General, Asistente del servicio de Cirugía General. 


\section{INTRODUCCIÓN}

Los quistes hepáticos simples son poco frecuentes, afectan entre el 5-10\% de la población ${ }^{(1)}$. La incidencia es mayor en mayores de 50 años, con una relación mujer/hombre 1,5/1 (2).

El quiste hepático simple es una malformación biliar ${ }^{(2)}$. Debido a que durante la embriogénesis, se desarrolla un número excesivo de conductos biliares intrahepáticos ${ }^{(3)}$, y en donde existe una falla de estos conductos biliares intrahepáticos para conectarse con los conductos biliares extrahepáticos $^{(4)}$.

Es decir, pierden su comunicación con el árbol biliar, ya sea por obstrucción ductal o por una hiperplasia inflamatoria de los conductos, y consecuentemente se dilatan para formar los quistes hepáticos simples, con la consiguiente retención de líquido en su interior. Es por eso que el quiste solo contiene agua y electrolitos sin contenido de sales biliares y bilirrubina ${ }^{(5)}$. Otra causa del quiste hepático simple es el trauma abdominal que causa la extravasación de bilis por fuera del árbol biliar ${ }^{(6)}$.

Microscópicamente, los quistes simples están revestidos por una sola capa de células epiteliales cuboides o columnares, semejantes a las células epiteliales biliares ${ }^{(2)}$. Macroscópicamente, son generalmente esféricos u ovoides, uniloculares y sin septos; pueden ser pequeños $(1 \mathrm{~cm}) 0$ grandes $(20 \mathrm{~cm})^{(7,8)}$. No tienen potencial maligno ${ }^{(9)}$. Los grandes quistes pueden comprimir hígado, induciendo a la atrofia regional y a veces a la compensación hipertrófica contralateral ${ }^{(7)}$. El $50 \%$ de los quistes son solitarios, pero pueden ser múltiples, que a diferencia de la enfermedad poliquística hepática, ocupan menos del $50 \%$ del hígado ${ }^{(10)}$. Se localizan sobre todo en el lóbulo derecho del hígado ${ }^{(11)}$.

La ecografía es el método de elección para el diagnóstico de estos quistes ${ }^{(8)}$. La tomografía y resonancia magnética pueden ser útiles para diferenciarlos de otras anormalidades ${ }^{(12)}$. Los criterios ultrasonográficos para el diagnóstico de quiste hepático simple son: imagen anecoica (es decir, cavidad llena de fluido), sin tabiques, bordes lisos afilados, fuertes ecos de la pared posterior (lo que indica una interfaz de fluido/tejido bien definido), esférica o de forma ovalada y una acentuación relativa de los ecos más allá del quiste en comparación con ecos en una profundidad similar transmitida a través de tejido hepático normal adyacente ${ }^{(13)}$.

En la tomografía sin contraste, los quistes hepáticos son lesiones de densidad de agua, con márgenes claramente definidos y paredes lisas y delgadas. Normalmente carecen de septos y no muestran niveles de detritus de fluidos, nodularidad mural o calcificación de la pared ${ }^{(14)}$. En la tomografía con contraste no hay realce del contenido del quiste $^{(15)}$.

La mayoría de los quistes son asintomáticos, sin embargo, el $5 \%$ de los pacientes desarrollan síntomas, a veces debido a complicaciones y que requerirán intervención ${ }^{(1)}$.
Actualmente no existe literatura con respecto al estudio de la litiasis vesicular concomitante en los pacientes con quistes hepáticos simples, así como poco conocimiento de los volúmenes de los quistes y los segmentos hepáticos comprometidos en su totalidad en los quistes múltiples.

El objetivo del presente trabajo fue conocer si existe relación entre el quiste hepático simple múltiple y la presencia de litiasis, porque la literatura refiere que el quiste hepático simple es una malformación de la vía biliar intrahepática ${ }^{(2)}$ y esto podría afectar la vesícula. El conocimiento de esta relación nos permitirá la prevención de colelitiasis futuras en pacientes con quistes hepáticos simples.

\section{MATERIALES Y MÉTODOS}

Se realizó un estudio observacional, retrospectivo y transversal, en el Servicio de Cirugía General del Hospital III de Emergencias Grau (EsSalud).

La unidad de análisis fue el paciente con diagnóstico de quiste hepático simple mediante ecografía y/o tomografía, además de pruebas serológicas para descarte de quiste hidatídico. Se realizó un muestreo no probabilístico por conveniencia de tipo accidental, considerando a todos los pacientes con diagnóstico de quiste hepático simple entre los años 2012-2017, y registrados en la oficina de estadística del Hospital Grau. El tamaño de la muestra fue de 50 pacientes. El instrumento de recolección de datos fue una ficha de datos con alternativas cerradas y abiertas, utilizando la técnica de inspección de registros de datos de estadística, historias clínicas e imágenes para la elaboración de una base de datos.

Los criterios de inclusión fueron diagnóstico ecotomográfico de quiste hepático simple e historia clínica con datos completos requeridos en la ficha de datos; y los criterios de exclusión fueron pacientes pediátricos, diagnóstico serológico de quiste hidatídico, quistes simples complicados, y enfermedad poliquística hepática del adulto. Se utilizó medidas de tendencia central, de posición, de dispersión, análisis de correlación bivariada y chi-cuadrado. Se realizó el procesamiento y tabulación de datos mediante el programa de IBM SPSS Statistics 19.0.

El presente estudio contó con autorización del comité de ética del Hospital III de Emergencias Grau (EsSalud).

\section{RESULTADOS}

Todos los pacientes fueron mayores de 40 años de edad, entre los 50 a 87 años, rango de 37 , con una media de 66,74 años, moda de 69 años, siendo el grupo etario entre 6170 años el más frecuente con $32 \%$, seguido por el grupo 51-60 años con $28 \%$; de esta manera, más del $60 \%$ de los pacientes estuvieron entre los 51-70 años de edad. El cuartil Q1 fue de 57,50, el cuartil Q2 o mediana fue de 67 y el cuartil Q3 fue de 73,25 (Figura 1). 


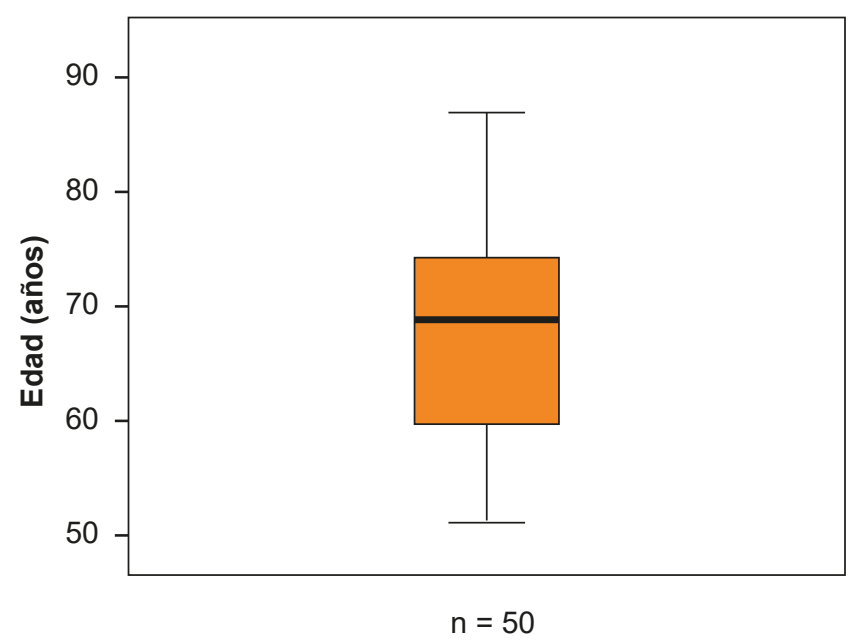

Figura 1. Diagrama de Caja de las Edades de los pacientes con quiste hepático simple

El género femenino fue el más frecuente con $92 \%$, existiendo una relación $11: 1$ con respecto al género masculino. La mayoría de los quistes hepáticos simples $(87,5 \%)$ tuvieron un volumen menor a $200 \mathrm{cc}$, pero hubo un $4,2 \%$ en que el volumen fue mayor a $1001 \mathrm{cc}$, con una media de $87,58 \mathrm{cc}$, moda de 0,16 cc, un volumen mínimo de 0,16 cc y un máximo de 1255 cc. Con una desviación estándar de 222,51 cc.

Un $52 \%$ de los quistes fueron únicos, seguidos por un 38 $\%$ que fueron dos, el número máximo encontrado fue de 5 quistes (2\%).

Se realizó un análisis de correlación bivariada entre la edad y el volumen del quiste simple, para determinar si existía una relación entre el tamaño del quiste y la edad, se tomó en cuenta el quiste de mayor tamaño en caso de los quistes múltiples, utilizando el Coeficiente de correlación lineal de Pearson, se encontró una correlación de 0,035, que representó una muy débil correlación positiva entre estas dos variables.

La totalidad de los quistes simples se localizaron en un 55,4 $\%$ en el lóbulo derecho. En cuanto a la localización de los quistes por segmento hepático, se observó que cuando se contabilizó solo el quiste de mayor tamaño en los casos de quistes múltiples, de los 50 quistes contabilizados, la mayoría se localizaron en el segmento hepático $V$ con $18 \%$, seguido por los segmentos II, IV y VII con $16 \%$.

Pero cuando se consideró la totalidad de los quistes hepáticos simples, grandes y pequeños (81 quistes), la localización más frecuente fue en el segmento II con 17,3 $\%$, seguido por los segmentos IV, V y VII con $16 \%$. Solo hubo 1 quiste ubicado en el segmento I (Tabla 1).

Tabla 1. Prueba de muestras emparejadas en Trujillo y Chimborazo

$\begin{array}{ccc}\text { Segmento hepático } & \text { Frecuencia } & \% \\ \text { II } & 1 & 1,2 \\ \text { III } & 14 & 17,3 \\ \text { IV } & 8 & 9,9 \\ \text { V } & 13 & 16,0 \\ \text { VI } & 13 & 16,0 \\ \text { VII } & 9 & 11,1 \\ \text { VIII } & 13 & 16,0 \\ \text { Total } & 10 & 12,3\end{array}$


El $56 \%$ de los quistes hepáticos simples se encontraron en hígados normales, seguido por un $36 \%$ en hígados con esteatosis (Tabla 2).

Tabla 2. Tipo de parénquima hepático en los pacientes con quiste hepático simple

\begin{tabular}{ccc} 
Parénquima hepático & Frecuencia & $\%$ \\
\hline Normal & 28 & 56,0 \\
Esteatosis & 18 & 36,0 \\
Hepatopatía crónica difusa & 4 & 8,0 \\
\hline Total & $\mathbf{5 0}$ & 100 \\
\hline
\end{tabular}

No se encontró dilatación de vías biliares en el $100 \%$ de los casos, incluso en los quistes mayores de 1000 cc de volumen.

El 83,78 \% de los pacientes con quiste hepático simple no presentaron litiasis vesicular, sólo el 16,21 \% lo presentó. En este caso solo se tomaron en cuenta los pacientes con quistes hepáticos simples con vesícula, porque 13 de los 50 pacientes eran colecistectomizados. En la revisión de sus historias clínicas de estos 13 pacientes, no estaba registrada la causa de la colecistectomía, ni la ecografía previa a la colecistectomía. Por lo cual se excluyó del estudio a los 13 pacientes colecistectomizados (Tabla 3).

Tabla 3. Colelitiasis en los pacientes con quiste hepático simple

\begin{tabular}{ccc} 
Colelitiasis & Frecuencia & $\%$ \\
Sí & 6 & 16,21 \\
No & 31 & 83,78 \\
Total & 37 & 100 \\
\hline
\end{tabular}

Si se tomara en cuenta los pacientes colecistectomizados como pacientes con litiasis, aun así un $62 \%$ de los pacientes no presentarían litiasis vesicular.

Se investigó si existía relación entre el número de quistes (único o múltiple) y la presencia o ausencia de litiasis vesicular, para lo cual se realizó la prueba de chi-cuadrado, con un resultado de $0,828(\mathrm{p}<0,05)$, por lo cual estas dos variables no estuvieron asociadas, fueron independientes (Tabla 4).

Tabla 4. Tabla de contingencia colelitiasis - quiste hepático simple múltiple

\begin{tabular}{crrrrrr} 
Colelitiasis & & Quiste múltiple & & Frecuencia & $\%$ \\
Sí & Sí & $\%$ & No & $\%$ & & 16,21 \\
No & 3 & 8,10 & 3 & 8,10 & 6 & 83,78 \\
Total & 17 & 45,94 & 14 & 37,83 & 31 & $\mathbf{3 7}$ \\
\hline
\end{tabular}




\section{DISCUSIÓN}

El diagnóstico de quiste hepático simple se realizó por imágenes porque la gran mayoría de quistes fueron asintomáticos y no tuvieron indicación de cirugía, solo 6 quistes fueron sometidos a cirugía y fueron confirmados los diagnósticos mediante anatomía patológica.

El presente trabajó evidenció que los quistes simples son más frecuentes en personas a partir de la cuarta década, como lo presentan otros trabajos ${ }^{(5,16,17)}$; pero que también puede aparecer a temprana edad ${ }^{(11)}$, cosa que no se presentó en este estudio.

El género femenino fue predominante, como lo menciona también Cuenca ${ }^{(5)}$ y Manterola ${ }^{(17)}$, con una relación mujer/ hombre muy alta, parecida a lo manifestado por $\mathrm{Kim}^{(7)} \mathrm{con}$ $8 / 1$, a diferencia de otros trabajos que mencionan una relación de 1,5:1 ${ }^{(6)}$.

Los quistes fueron generalmente únicos como lo describe también otros autores ${ }^{(7,16)}$. Tomaz ${ }^{(6)}$ manifiesta que hay literatura que indica que el número de quistes aumenta con la edad porque a mayor edad hay mayor incidencia de estasis en las vías biliares y obstrucción que conlleva a la formación de más quistes, algo que no se evidenció con el presente trabajo.

La localización frecuente del quiste simple en el lóbulo hepático derecho es descrito en varios trabajos de investigación ${ }^{(5)}$, pero hay otros como Stănescu ${ }^{(18)}$ que refieren que es en el lóbulo izquierdo (75 \%).

Existen muy pocas publicaciones sobre la localización de los quistes simples a nivel de segmentos hepáticos, como lo describe Tomaz ${ }^{(6)}$ quien indica que los segmentos hepáticos más afectados fueron los IVa (23\%) y VI (17 \%), lo que no coincidió no los hallazgos del presente estudio.

No hay trabajos de investigación sobre los volúmenes de los quistes hepáticos simples, ni el conteo completo de todos los quistes en el hígado afectado, sean grandes o pequeños.

En la mayoría de los trabajos mencionan solo el diámetro mayor del quiste, con variados promedios que van desde $0,2 \mathrm{~cm}$ hasta $16 \mathrm{~cm}^{(6,17,18)}$.

Díaz et al ${ }^{(13)}$ menciona que los quistes hepáticos simples alcanzan tamaños gigantes casi exclusivamente en los mayores de 50 años de edad, lo que coincidió con el presente trabajo, con los 2 casos de quistes con volúmenes mayores de $1000 \mathrm{cc}$.

Se evidenció que los quistes simples se encontraron con más frecuencia en hígados con parénquima normal, similar a lo que encontró Horta et al ${ }^{(16)}$ que encontró en hígado normal en un $27 \%$ de los casos, seguido de hígado con esteatosis con $12,9 \%$.

La frecuencia de presentación de la colelitiasis en pacientes con quistes hepáticos simples encontrada en el presente estudio es similar a la encontrada en la población en general que tiene una prevalencia de $10-15 \%{ }^{(19)}$, con lo que no hay una diferencia significativa entre ambas.

El no evidenciar una relación entre la presencia del quiste hepático simple múltiple y la litiasis vesicular quizás se deba a que las vías biliares intrahepáticas y la vesícula biliar tienen orígenes embriogénicos diferentes, porque las vías biliares intrahepáticas se forman a partir de la yema craneal del divertículo hepático y la vesícula biliar a partir de la yema caudal ${ }^{(20)}$. Y esto podría ser causa para la ausencia de esta relación.

En conclusión, la mayoría de quistes hepáticos simples fueron únicos, localizados en el lóbulo derecho, pero con mayor frecuencia en el segmento II. Los quistes hepáticos simples se encontraron generalmente en hígados con parénquima normal, sin colelitiasis, ni dilatación de vías biliares. No hubo correlación entre la edad y el volumen del quiste simple. No se evidenció una relación entre la presencia del quiste hepático simple múltiple y la litiasis vesicular.

\section{REFERENCIAS BIBLIOGRÁFICAS}

1. Macutkiewicz C, Plastow R, Chrispijn M, Filobbos R, Ammori BA, Sherlock DJ, et al. Complications arising in simple and polycystic liver cysts. World journal of hepatology. 2012;4(12):406. Disponible en: https://www.ncbi.nlm.nih. gov/pmc/articles/PMC3554807/

2. Macedo F. Current management of noninfectious hepatic cystic lesions: A review of the literature. World J Hepatol. 2013; 5(9): 462-469. Disponible en: https://www.ncbi.nlm. nih.gov/pmc/articles/PMC3782683/

3. Ueda J, Yoshida H, Taniai N, Mineta S, Kawano Y, Uchida E. Case Reports. A Case of Spontaneous Rupture of a Simple Hepatic Cyst. J Nippon Med Sch. 2010; 77(3): 181-185. Disponible en: https://www.jstage.jst.go.jp/article/ jnms/77/3/77_3_181/_article/-char/ja/

4. Lantinga M, Gevers T, Drenth J. Evaluation of hepatic cystic lesions. World J Gastroenterol. 2013; 19(23): 3543-3554. Disponible en: https://www.ncbi.nlm.nih.gov/pmc/articles/ PMC3691048/

5. Cuenca O, Ramirez J, Martinez D, Pfingst C, Morel E. Caso clínico. Manejo de los quistes hepáticos en el Servicio de Cirugía General del Hospital Nacional (Itauguá). Rev. Nac. (Itauguá). 2013; 5(2): 49-50. Disponible en: http://scielo.iics. una.py/pdf/hn/v5n2/v5n2a09.pdf

6. Tomaz B, Rios L, Prando P, Franchi T, Silveira P, D Ippolito $G$. Prevalence of simple liver cysts and hemangiomas in cirrhotic and non-cirrhotic patients submitted to magnetic resonance imaging. Radiol Bras. 2013; 46(4): 203-208. Disponible en: http://www.scielo.br/scielo.php?script=sci_ arttext\&pid=S0100-39842013000400203

7. Kim S-R, Lee D-S, Park I-Y. Managements of simple liver cysts: ablation therapy versus cyst unroofing. Korean J Hepatobiliary Pancreat Surg. 2012; 16(4): 134-137. Disponible en: https:// www.ncbi.nlm.nih.gov/pmc/articles/PMC4574997/

8. De Santibañes E, Mazza O, Quiñonez E. Quistes hepáticos no parasitários. Cirugía Digestiva. 2009; 4(409): 1-10. Disponible en: http://sacd.org.ar/cnueve.pdf

9. Motta Ramírez GA, Alonso Blancas E, Chirino Sprung RA, Irais González-Merino L. Characterización de lesiones hepáticas focales con tomografía computada multidetector. Anales de 
Radiologia, Mexico. 2012;11(1): 46-58. Disponible en: http:// www.medigraphic.com/pdfs/anaradmex/arm-2012/arm121g. pdf

10. Ramia JM, de La Plaza R, Figueras J, García-Parreño J. Tumores hepáticos quísticos benignos no parasitarios. Cirugía Española. 2011;89(9):565-73. Disponible en: http://www. sciencedirect.com/science/article/pii/S0009739X11002132

11. Banerjee R, Lakhoo K. Case report. A rare large symptomatic simple hepatic cyst. BMJ Case Rep. 2013; 1-3. Disponible en: http://casereports.bmj.com/content/2013/bcr-2013200094.abstract

12. Dinc Dinc B, Mesci A, Enver S, Oskay A. A Giant Simple Liver Cyst That Caused Increases in Serum CA 19-9 and CA 15-3 Levels J Clin Med Res. 2014; 6(6): 487-489. Disponible en: https://www.ncbi.nlm.nih.gov/pmc/articles/PMC4169093/

13. Díaz-Girón Gidi A, González-Chávez MA, González HermosilloCornejo D, Vélez-Pérez FM, Espino-Urbina L, Visag-Castillo $\mathrm{V}$, et al. Quiste hepático gigante simple. Reporte de un caso y revisión de la literatura. Médica Sur. 2016;22(2):94 8.Disponible en: http://new.medigraphic.com/cgi-bin/ resumen.cgi?IDARTICULO $=67279$

14. Vachha B, Sun M, Siewert B, Eisenberg R. Cystic Lesions of the Liver. AJR. 2011; 196(4): W335-W366. Disponible en: https:// www.ajronline.org/doi/full/10.2214/AJR.10.5292

15. Aza D. Determinar la prevalencia de lesiones hepáticas por medio de tomografía simple y contrastada de abdomen, en pacientes de 30 a 60 años de edad en la clínica de especialidades oftálmica en el período de Julio a Diciembre del 2014 [Tesis Licenciatura]. Quito, Ecuador: Universidad central del Ecuador; 2015

16. Horta G, López M, Dotte A, Cordero J, Chesta C, Castro A, et al. Lesiones focales hepáticas benignas: un hallazgo frecuente a la tomografía computada. Rev méd Chile. 2015;143(2):197-202. Disponible en: https://scielo.conicyt. $\mathrm{cl} /$ scielo.php?pid=S0034-98872015000200007\&script=sci_ arttext\&tlng=pt

17. Manterola C. Resultados de la Cirugía Laparoscópica en Quistes Hepáticos No Parasitarios. Int. J. Morphol. 2013; 31(3): 1116-1119. Disponible en: https://scielo.conicyt.cl/ scielo.php?pid=S0717-95022013000300054\&script=sci_arttext

18. Stănescu C, Păduraru D, Cirimbei C, Brătucu E. The laparoscopic management of simple hepatic cysts. J Med Life. 2015; 8(3): 342-345. Disponible en: https://pdfs.semanticscholar.org/ e3b6/826f26259b31654329188121fd560f20382b.pdf

19. Goral V. Gallstone Etiopathogenesis, Lith and Mucin Genes and New Treatment Approaches. Asian Pac J Cancer Prev. 2016; 17(2): 467-471. Disponible en: http://www.dbc.fmed.edu.uy/ sites/www.dbc.fmed.edu.uy/files/Residentes/Gallstone\%20 Ethiphatogenesis\%2C\%20Lith\%20and\%20Mucin\%20Genes\%20 and\%20New\%20Treatment\%20approaches.pdf

20. Roa I, Meruane M. Desarrollo del aparato digestivo. Int. J. Morphol. 2012; 30(4): 1285-1294. Disponible en: https: / / scielo.conicyt.cl/scielo.php?pid=S0717. 95022012000400006\&script=sci_arttext\&tlng=en

\section{Fuentes de financiamiento:}

Autofinanciado por el autor

Conflictos de interés:

El autor declara no tener ningún conflicto de interés

\section{Correspondencia:}

Hubert James Mendoza Rojas

Dirección: Avenida Grau №351, Cercado de Lima. Lima, Perú.

Teléfono: 992758186

Correo electrónico: hubertjames86@hotmail.com

Recibido: 23 de octubre de 2017

Evaluado: 25 de octubre de 2017

Aprobado: 10 de diciembre de 2017

(c) La revista. Publicado por Universidad de San Martín de Porres, Perú. (c) Bу Licencia de Creative Commons Artículo en acceso abierto bajo términos de Licencia Creative Commons Atribución 4.0 Internacional. (http://creativecommons.org/licenses/by/4.0/)

ORCID iDs

Hubert James Mendoza Rojas (iD http://orcid.org/0000-0001-5880-9775 